The Journal of Laryngology \& Otology

http://journals.cambridge.org/JLO

Additional services for The Journal of Laryngology \& Otology:

Email alerts: $\underline{\text { Click here }}$

Subscriptions: $\underline{\text { Click here }}$

Commercial reprints: Click here

Terms of use : $\underline{\text { Click here }}$

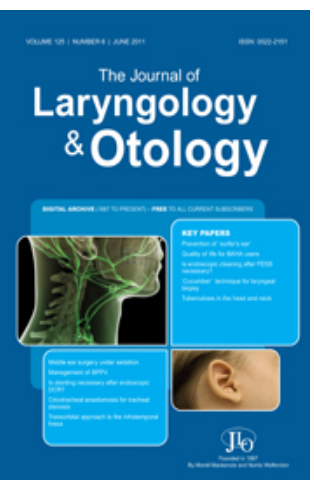

\title{
Prospective, randomised controlled trial comparing intense endoscopic cleaning versus minimal intervention in the early post-operative period following functional endoscopic sinus surgery
}

\author{
J M Fishman, S Sood, M Chaudhari, P Martinez-Devesa, L Orr and D Gupta
}

The Journal of Laryngology \& Otology / Volume 125 / Issue 06 / June 2011, pp 585 - 589

DOI: 10.1017/S0022215111000508, Published online: 28 March 2011

Link to this article: http://journals.cambridge.org/abstract_S0022215111000508

How to cite this article:

J M Fishman, S Sood, M Chaudhari, P Martinez-Devesa, L Orr and D Gupta (2011). Prospective, randomised controlled trial comparing intense endoscopic cleaning versus minimal intervention in the early post-operative period following functional endoscopic sinus surgery. The Journal of Laryngology \& Otology, 125, pp 585-589 doi:10.1017/S0022215111000508

Request Permissions : $\underline{\text { Click here }}$ 


\title{
Prospective, randomised controlled trial comparing intense endoscopic cleaning versus minimal intervention in the early post-operative period following functional endoscopic sinus surgery
}

\author{
J M FISHMAN, S SOOD, M CHAUDHARI, P MARTINEZ-DEVESA, L ORR, D GUPTA \\ Department of Otolaryngology, The Great Western Hospital, Swindon, Wiltshire, UK
}

\begin{abstract}
Objective: There is currently no standardised management protocol following functional endoscopic sinus surgery. This study assessed frequent endoscopic cleaning versus minimal intervention in the early post-operative period following such surgery.

Study design: Prospective, randomised controlled, single-blinded, within-subject trial involving 24 patients with bilateral chronic rhinosinusitis undergoing bilateral functional endoscopic sinus surgery.

Main outcome measure: The primary outcome measure was ethmoid cavity healing, based on endoscopic appearance, graded using a modified Lund-MacKay endoscopic score.

Secondary outcome measure: Lund-MacKay symptom score before and after surgery.

Results: There was no overall statistically significant difference between the two groups $(p=0.37)$. Subgroup analysis revealed a significant effect of regular suction clearance on adhesions at three months $(p=0.048)$, but not on oedema, polyps, granulation, discharge or crusting.

Conclusion: There is no evidence from this study to support frequent endoscopic cleaning in the early postoperative period after functional endoscopic sinus surgery. Less intensive post-operative management is recommended, resulting in decreased patient morbidity and fewer post-operative follow-up appointments.
\end{abstract}

Key words: Post-Operative Complications; Post-Operative Care; Sinusitis; Endoscopy; Wound Healing

\section{Introduction}

There is no standardised management protocol following functional endoscopic sinus surgery (FESS). There is wide variation between surgeons, and also within the same surgeon's practice at different times. While Wigand has advised intense post-operative cleaning up to one week, Lund and MacKay have described cavity cleaning 5-10 days after surgery and then weekly thereafter until adequate healing occurs. ${ }^{1,2}$

Frequent follow up and vigorous post-operative cleaning is uncomfortable for patients, time-consuming and costly. The long term results of FESS with minimal post-operative follow up were reviewed in 1996 by Ryan et al.; they found success rates close to those of major centres practising vigorous follow-up cleaning. ${ }^{3}$ The role of frequent cleaning has also been questioned by Fernandes, who in 1999 quoted good results in children managed with minimal follow up (due to problems with cleaning under local anaesthesia). ${ }^{4}$

In our hospital, patients undergoing FESS are typically followed up six weeks after surgery. Nasal cavity debridement is carried out at this time if required.

Since most healing takes place in the first six weeks following FESS, the present study was designed to establish whether patients should be followed up and debrided more frequently in the early post-FESS period.

\section{Materials and methods}

Study design

This study was a prospective, randomised, controlled, single-blinded, within-subject trial (Figure 1). The study was designed in line with the latest Consort Group guidelines. 5 


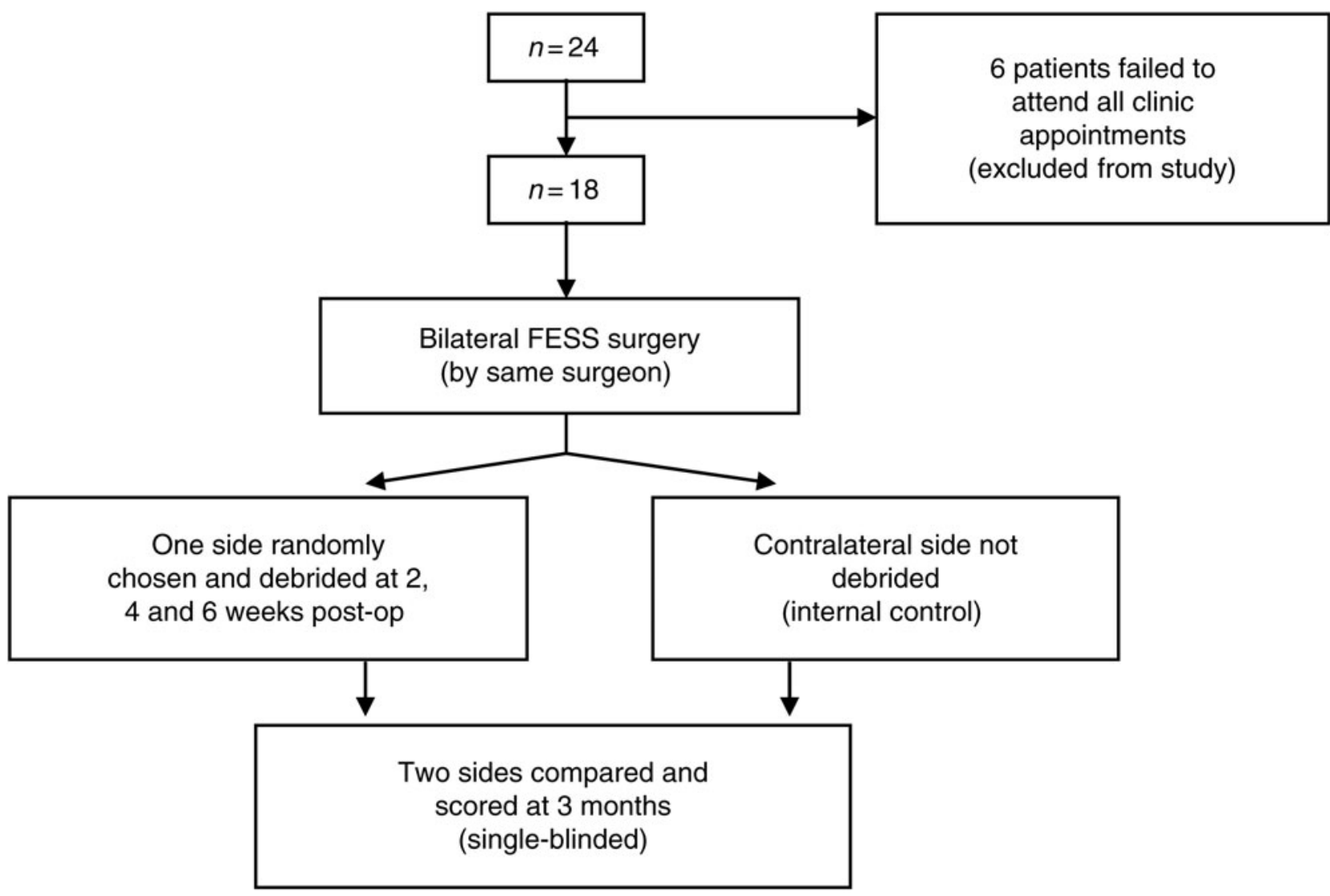

FIG. 1

Study design. FESS = functional endoscopic sinus surgery; post-op = post-operatively.

\section{Ethical considerations}

Local research ethics committee approval was obtained from the Swindon and Marlborough National Health Service Trust.

Patients undergoing FESS at The Great Western Hospital were approached to participate in the study, and their consent obtained before enrolment.

\section{Participants}

Twenty-four patients were enrolled in the study (12 men and 12 women; mean age 55 years; age range 22-77 years). Inclusion and exclusion criteria are given in Table I.

\section{TABLE I}

\section{STUDY INCLUSION AND EXCLUSION CRITERIA}

Inclusion criteria

Bilateral FESS

Persistent, bilateral, symmetrical CRS*

Exclusion criteria

Revision FESS cases

Previous rhinological surgery

Unilateral disease

Age $<16$ years

Unwilling to participate in study

Missed follow-up appointments

*Unresponsive to medical management and confirmed by computed tomography. FESS = functional endoscopic sinus surgery; $\mathrm{CRS}=$ chronic rhinosinusitis

\section{Surgery}

All patients with bilateral chronic rhinosinusitis, persisting despite optimal medical management, underwent bilateral surgery performed by the same surgeon (DG).

All patients underwent pre-operative computed tomography (CT) to confirm the clinical diagnosis and to assess the extent of disease. Based on the CT, each sinus group (maxillary, anterior ethmoids, posterior ethmoids, sphenoid and frontal) was graded between 0 and 2 according to the Lund-MacKay CT staging system, where $0=$ no abnormality, $1=$ partial opacification and $2=$ complete opacification. ${ }^{6,7}$ The ostiomeatal complex was scored as 0 (not obstructed) or 2 (obstructed). Each side was considered separately, giving a total score of 0 to 12 .

All patients underwent FESS involving at least a middle meatal antrostomy, with antero-posterior ethmoidectomy and middle turbinate quilting to the septum. Nasal packs, if required, were placed bilaterally.

All patients received standardised post-operative care, consisting of regular saline douches and three weeks of Betnesol ${ }^{\circledR}$ nasal drops, followed by a longterm intranasal steroid spray.

Follow-up clinic appointments were arranged at two weeks, four weeks, six weeks and three months postoperatively. 


\section{Randomisation and intervention}

Each patient acted as their own control (i.e. withinsubject design).

One side of the nose was chosen randomly and cleaned during the two, four and six week follow-up appointments (this was the treatment (or intervention) side). Cleaning was carried out under topical local anaesthesia, using suction and a $0^{\circ}$ nasendoscope.

The contralateral side was left untouched (this was the control side).

Randomisation of the side selected for frequent cleaning (i.e. the intervention side) was carried out by allocation concealment, using sequentially numbered opaque, sealed envelopes indicating the right or left nostril. The envelope was opened at the first followup clinic appointment, at two weeks.

\section{Blinding}

A different surgeon, who was blinded to the side of the intervention, was asked to compare and score the two sides at three months post-surgery, based on their endoscopic appearance and using a modified Lund-MacKay endoscopic score, in order to determine the effect of regular nasal debridement on sinonasal mucosal healing.

\section{Outcome measures}

The primary outcome measure was ethmoid cavity healing, assessed endoscopically using six parameters (i.e. oedematous mucosa, polypoid mucosa, granulations, mucopurulent discharge, adhesions and crusting). Each of the six parameters was graded as 0 (absent), 1 (mild), 2 (moderate) or 3 (severe), according to its endoscopic appearance. Thus, each side could have a minimum score of 0 and a maximum score of 18 .

The secondary outcome measure comprised comparison of symptom scores before and after surgery. Symptoms were assessed by the patient on a visual analogue score of 0 to 10 , according to the Lund-MacKay symptoms score, with 0 indicating no symptoms and 10 indicating the most severe symptoms, for nasal obstruction, sense of smell, headache and facial pain. ${ }^{6,7}$

\section{Statistical and power analysis}

Assuming 16 patients per group and a significance of 5 per cent, we calculated that the study would have a power of 80 per cent to detect a mean difference of 2 points between the two groups (considered to be a clinically important difference). With each patient acting as their own control, this calculation indicated that a total group size of at least 16 patients would be sufficient to identify any significant detectable difference between the two sides.

Study results were found to be normally distributed, and were analysed accordingly using a paired twosample Student's $t$-test. The Statistical Package for the Social Sciences version 15 software program (SPSS Inc, Chicago, Illinois, USA) was utilised. Results were analysed using a per protocol analysis. Differences were regarded as statistically significant if the $p$ value was less than 0.05 . For the main outcome measures, 95 per cent confidence intervals (CIs) were calculated.

\section{Results and analysis}

Twenty-four patients were recruited into the study. Six patients failed to attend all their post-operative followup appointments and were thus excluded from the study (Table I), leaving 18 patients for analysis (Figure 1). The reasons given for non-attendance were inconvenient appointments (two patients) and procedure discomfort (four patients). In the 18 patients analysed in the study, clinic attendance was 100 per cent at two weeks, four weeks, six weeks and three months following surgery.

There was no significant difference in Lund-MacKay CT scores between the two sides, which otherwise may have biased the results (mean right score $=7.44$, mean left score $=6.56 ; p=0.41$, $t$-test, paired two sample for means).

Table II shows patients' modified Lund-MacKay endoscopic scores, without taking matching into account. Within-subject differences are displayed in Figures 2 and 3. Although the mean score was lower on the intervention side (mean \pm standard error of the mean (SEM) $1.50 \pm 0.90)$, compared with the control side (mean \pm SEM $2.94 \pm 1.17$; mean difference $1.44,95$ per cent $\mathrm{CI}$ of the difference -1.90 to $4.79)$, this difference was not statistically significant ( $p=0.37, t$-test, paired two sample for means, $n=$ 18; Figure 2). In addition, there was no statistically significant difference between the two sides with respect to oedema $(p=0.15)$, polyps $(p=0.84)$, granulations $(p=0.87)$, discharge $(p=0.87)$ and crusting $(p=$ $0.46)$. However, subgroup analysis revealed a statistically significant difference in adhesion scores between the two sides at three months $(p=0.048$, Student's $t$-test; Figure 3).

\begin{tabular}{lllll}
\multicolumn{5}{c}{ TABLE II } \\
MODIFIED LUND-MACKAY ENDOSCOPIC APPEARANCE \\
SCORE: UNMATCHED FINDINGS \\
\hline Parameter, at 3 mth & \multicolumn{4}{c}{ Pts with score $(n)$} \\
\cline { 2 - 5 } & 0 & 1 & 2 & 3 \\
\hline Oedema (+) & 15 & 2 & 0 & 1 \\
Oedema (-) & 12 & 2 & 1 & 3 \\
Polyps (+) & 15 & 2 & 0 & 1 \\
Polyps (-) & 14 & 3 & 1 & 0 \\
Granulations $(+)$ & 15 & 2 & 0 & 1 \\
Granulations $(-)$ & 15 & 1 & 0 & 2 \\
Adhesions (+) & 18 & 0 & 0 & 0 \\
Adhesions (-) & 14 & 1 & 0 & 3 \\
Discharge $(+)$ & 15 & 1 & 1 & 1 \\
Discharge (-) & 16 & 0 & 0 & 2 \\
Crusting (+) & 15 & 2 & 1 & 0 \\
Crusting (-) & 15 & 1 & 0 & 2 \\
\hline
\end{tabular}

Modified Lund-MacKay endoscopic score: $0=$ absent; $1=$ mild; $2=$ moderate; $3=$ severe. Mth $=$ months; pts $=$ patients; $+=$ treatment side; $-=$ control side. 


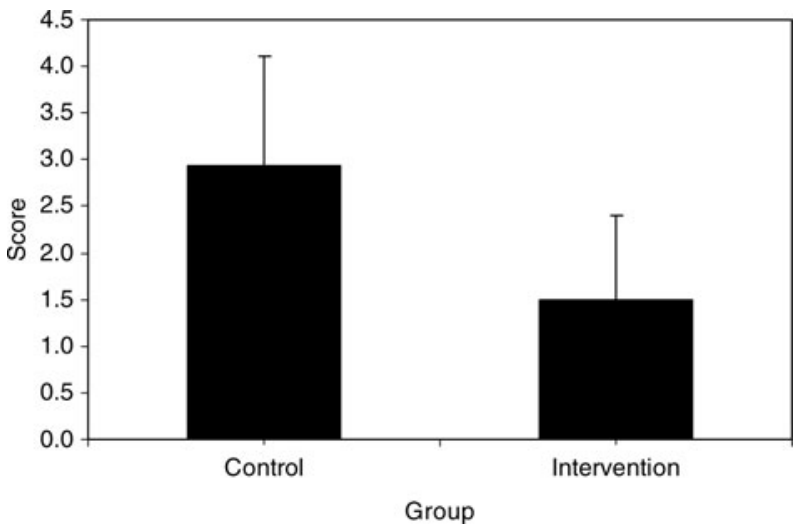

FIG. 2

Overall mean modified Lund-MacKay endoscopic scores in the intervention and control groups at three months. No statistically significant difference was seen $(p=0.37)$. Results are presented as mean \pm SEM

There was a significant improvement in post- versus pre-operative symptom scores for nasal obstruction ( $p=0.0008, t$-test, paired two sample for means), sense of smell $(p=0.0087)$, and facial pains and/or headaches $(p=0.0058)$. Of the 18 patients analysed, five ( 27.8 per cent) reported that the debridement procedure caused pain or discomfort, even with the use of topical local anaesthetic spray.

\section{Discussion}

\section{Synopsis of key findings}

There was no statistically significant difference in the overall endoscopic appearance of the two sides at three months post-surgery $(p=0.37, t$-test, paired two sample for means, $n=18)$. Subgroup analysis revealed that regular suction clearance had a significant effect only on adhesions at three months $(p=0.048)$,

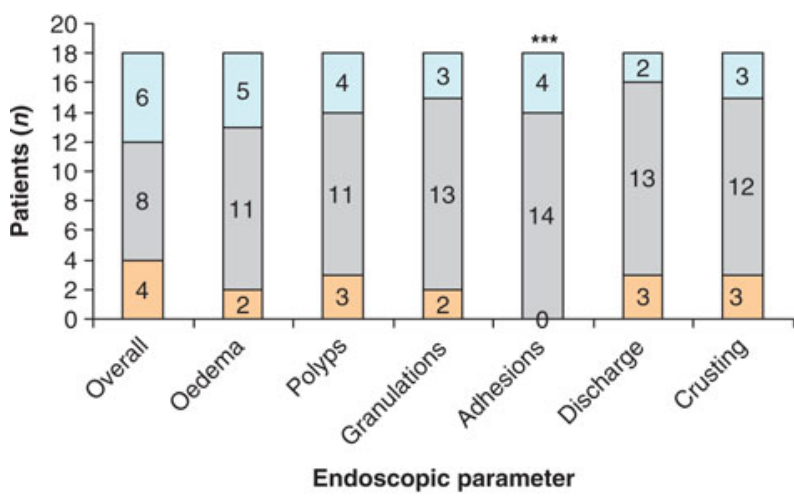

$\square$ Intervention better

$\square$ No difference

$\square$ Control better

FIG. 3

Analysis of differences between the intervention and control groups at three months, for the six endoscopic parameters. The only significant effect of regular suction clearance was on adhesions ( $p=$ 0.048). with no effect on oedema, polyps, granulations, discharge or crusting. It is possible that the observed effect on adhesions was secondary to the effect of quilting of the middle turbinate to the septum, rather than being due to regular suction clearance per se, although the middle turbinate was preserved in all cases and sutured to the septum bilaterally in the same manner. In any event, a larger study would be required to determine whether the observed effect on adhesions was reproducible.

\section{Comparison with other studies}

There is currently no standardised post-operative regime following FESS. Both the frequency and the optimal number of post-operative visits vary greatly between different endoscopic sinus surgeons. To our knowledge, the present study is the first randomised, controlled trial utilising a within-subject design to assess whether frequent endoscopic debridement of ethmoid cavities is effective in reducing post-operative signs, as assessed endoscopically.

Our findings are in agreement with those of Nilssen et al., who in their 2002 study were unable to demonstrate a significant benefit from post-operative FESS cavity debridement, at least with regard to cavity healing. ${ }^{8}$ However, they considered their study a pilot study, and stressed that further work was required to determine the optimal post-operative care for patients undergoing FESS. In addition, no attempt was made to separate the effects of frequent cavity debridement upon oedema, polyps, discharge, crusting and adhesions.

Ryan et al. and Fernandes have also questioned the value of regular post-operative cavity cleaning, although neither formally tested this using a randomised, controlled trial. ${ }^{3,4}$

Lee and Byun concluded that one-week intervals were the optimal frequency for post-operative debridement during the healing period after endoscopic sinus surgery; however, no statistically significant differences were reported between the groups as regards healing period and objective endoscopic findings. ${ }^{9}$ In addition, patients across groups were compared and a within-subject design was not utilised.

In a study of 90 patients, Kemppainen et al. concluded that, in terms of subjective recovery and healthcare costs, repeated debridement was not justified during the first post-operative week following FESS; this is in agreement with our study. ${ }^{10}$ However, at four weeks, significantly fewer cavities presented with nasal discharge in the intervention group compared with the control group. There were no other statistically significant differences in Lund-MacKay endoscopic appearance scores (including for adhesions).

The difference between Kemppainen and colleagues' results and those of the present study, with regard to adhesions and nasal discharge, may be attributed to differences in the frequency of post-operative follow up (Kemppainen et al. performed debridement 
only during the first post-operative week, while we performed it for up to six weeks) and study design (Kemppainen et al. did not blind their assessor, nor use a within-subject design).

In addition, and in agreement with our findings, Bugten et al. reported a decrease in the development of adhesions following the use of post-operative cleaning after FESS. ${ }^{11}$ However, they acknowledged that the procedure induced more post-operative nasal pain and discomfort. Such pain and discomfort was responsible for four of our patients failing to attend their follow-up appointments. In addition, five of our 18 patients (27.8 per cent) reported pain or discomfort during the procedure.

\section{Study strengths and limitations}

This was a prospective, randomised, single-blinded, within-subject study designed to minimise the number of variables complicating the main treatment comparison in question. The study used one surgeon, a standardised surgical technique on both sides of the nose and a standardised post-operative treatment regimen. In addition, all patients had bilateral, symmetrical sinus disease, and there was no significant difference in Lund-MacKay CT scores between the two sides of the nose (which may otherwise have biased results). Clinic attendance was 100 per cent at two, four and six weeks and three months postsurgery, since patients who missed post-operative clinic appointments were excluded from the study (Table I).

- In this study of functional endoscopic sinus surgery (FESS) follow-up protocols, 24 patients were randomised to receive either frequent endoscopic cleaning or minimal intervention

- No significant difference in endoscopic appearance or symptom scores was seen between the two groups

- Endoscopic cleaning is not justified during the early post-FESS period

- A less intensive post-FESS follow-up protocol results in decreased patient pain and discomfort and requires fewer follow-up appointments, while still achieving satisfactory sinonasal mucosal healing

We acknowledge a number of limitations to this study. In the first instance, the study contained a relatively small number of patients, although our a priori power analysis suggested that a group of only 16 patients would be sufficient to identify any significant difference. In addition, the duration of follow up was short (three months). Longer follow up (e.g. six or 12 months) would make for an interesting subsequent study, especially as regards whether the effect of regular suction clearance on adhesions is real and sustainable in the longer term.

Our study made no attempt to separate out chronic rhinosinusitis patients with and without polyps, since surgery was conducted in exactly the same manner in both groups. However, future studies may like to compare the effect of regular post-operative endoscopic cleaning in these two different patient subgroups.

\section{Conclusion and clinical applicability}

In terms of subjective recovery, objective endoscopic appearance and healthcare cost, repeated endoscopic cleaning (performed to achieve satisfactory sinonasal mucosal healing) is not justified during the early post-operative period following FESS. A less intense post-operative follow-up regime reduces patient pain and discomfort and requires fewer follow-up appointments.

\section{Acknowledgements}

We gratefully acknowledge all the patients who participated in this research. We also thank all the staff of the ENT outpatient department of The Great Western Hospital, Swindon, for making this study possible.

\section{References}

1 Wigand ME. Endoscopic Surgery of the Paranasal Sinuses and Anterior Skull Base. New York: Thieme, 1990

2 Lund VJ, MacKay IS. Outcome assessment of endoscopic sinus surgery. J R Soc Med 1994;87:70-2

3 Ryan RM, Whittet HB, Norval C, Marks NJ. Minimal follow-up after functional endoscopic sinus surgery. Does it affect outcome? Rhinology 1996;34:44-5

4 Fernandes SV. Postoperative care in functional endoscopic sinus surgery? Laryngoscope 1999;109:945-8

5 Schulz KF, Altman DG, Moher D, for the CONSORT Group. CONSORT 2010 statement: updated guidelines for reporting parallel group randomised trials. BMJ 2010;340:c332

6 Lund VJ, Mackay IS. Staging in rhinosinusitis. Rhinology 1993; 31:183-4

7 Lund VJ, Kennedy DW. Staging for rhinosinusitis. Otolaryngol Head Neck Surg 1997;117:S35-40

8 Nilssen EL, Wardrop P, El-Hakim H, White PS, Gardiner Q, Ogston S. A randomized control trial of post-operative care following endoscopic sinus surgery; debridement versus no debridement. J Laryngol Otol 2002;116:108-11

9 Lee JY, Byun JY. Relationship between the frequency of postoperative debridement and patient discomfort, healing period, surgical outcomes, and compliance after endoscopic sinus surgery. Laryngoscope 2008;118:1868-72

10 Kemppainen T, Seppa J, Tuomilehto H, Kokki H, Nuutinen J. Repeated early debridement does not provide significant symptomatic benefit after ESS. Rhinology 2008;46:238-42

11 Bugten V, Nordgard S, Steinsvag S. The effects of debridement after endoscopic sinus surgery. Laryngoscope 2006;116: 2037-43

Address for correspondence:

Mr Jonathan Fishman,

ENT Registrar \& Royal College of Surgeons of England Surgical

Research Fellow,

Department of Otolaryngology,

The Great Western Hospital, Swindon, Wiltshire SN3 6BB, UK

E-mail: jfishman@doctors.org.uk

Mr J Fishman takes responsibility for the integrity of the content of the paper

Competing interests: None declared 